\title{
Adding Prandial Insulin to Basal Insulin Plus Oral Antidiabetic Drugs in Chinese Patients with Poorly Controlled Type 2 Diabetes Mellitus: An Open-Label, Single-Arm Study
}

Yujing Jin $\cdot$ Xiaowei Sun $\cdot$ Xichen Zhao $\cdot$ Tiehong Zhu

Received: February 21, 2017 / Published online: March 27, 2017

(C) The Author(s) 2017. This article is an open access publication

\section{ABSTRACT}

Introduction: There is relatively little data from China on the efficacy and safety of adding prandial insulin to basal insulin plus oral antidiabetic drugs (OADs) in people with poorly controlled type 2 diabetes mellitus (T2DM). This study assessed the efficacy and safety of basal insulin dose optimization followed by the addition of prandial insulin in Chinese people with T2DM achieving suboptimal glycemic control with basal insulin and OADs.

Methods: In this open-label, single-arm study, adults with T2DM receiving basal insulin plus OADs underwent insulin dose optimization for 12 weeks. At week 12, subjects who achieved fasting blood glucose (FBG) $\leq 6.5 \mathrm{mmol} / \mathrm{L}$ but not $\mathrm{HbA} 1 \mathrm{c} \leq 7 \%$ added one injection of prandial insulin at the main meal for an additional 24 weeks. Endpoints included mean $\mathrm{HbA1c}$, the achievement rate of $\mathrm{HbA} 1 \mathrm{c} \leq 7 \%$, hypoglycemia, and other adverse events (AEs).

Results: A total of 120 subjects underwent basal insulin optimization; At week 12, 110 study subjects achieved FBG $\leq 6.5 \mathrm{mmol} / \mathrm{L}$, of whom $66 \mathrm{did}$

Enhanced content To view enhanced content for this article go to http://www.medengine.com/Redeem/ 06F7F060022F228A.

Y. Jin · X. Sun · X. Zhao · T. Zhu ( $₫)$

Tianjin Medical University General Hospital,

Tianjin 300052, China

e-mail: zhutiehong@tmu.edu.cn not achieve $\mathrm{HbA} 1 \mathrm{c} \leq 7 \%$ and therefore initiated prandial insulin. Three patients discontinued prandial insulin due to dissatisfaction with treatment outcome $(n=1)$, accidental injury $(n=1)$, or personal reasons $(n=1)$. After 24 weeks of basal-plus treatment, mean HbA1c significantly decreased $(8.06 \%$ to $7.17 \% ; p<0.001), 65.1 \%$ of subjects achieved $\mathrm{HbA1c} \leq 7 \%$, there was no change in FBG $(6.23-6.20 \mathrm{mmol} / \mathrm{L} ; p=0.118)$, and mean post-prandial blood glucose decreased (13.17-10.14 mmol/L; $p<0.001)$. During basalplus treatment, three individuals experienced hypoglycemia, and no significant change in the mean subject weight was observed (73.2 vs. $73.3 \mathrm{~kg} ; p=0.379$ ).

Conclusions: In people with T2DM who are achieving suboptimal glycemic control with basal insulin plus OADs, basal insulin dose optimization followed by the addition of prandial insulin improves glycemic control, is well tolerated, and is associated with a low incidence of hypoglycemia.

Keywords: Basal insulin; HbA1c; Insulin glargine; Prandial insulin; Type 2 diabetes

\section{INTRODUCTION}

Intensive control of blood glucose is crucial to minimizing the risk of disease-related complications in people with type 2 diabetes mellitus (T2DM) [1]. Achieving the glycemic control 
targets recommended by the American Diabetes Association (ADA) and other diabetes management guidelines, typically glycated hemoglobin (HbA1c) $\leq 7 \%[2,3]$, significantly reduces the incidence of micro- and macrovascular complications associated with T2DM $[4,5]$. However, due to the chronic and progressive nature of T2DM, achieving glycemic control becomes increasingly difficult as the disease advances.

If glycemic control cannot be achieved through lifestyle changes and maximum tolerated doses of one or more oral antidiabetic drugs (OADs), initiation of long-acting basal insulin, with dose titration based on fasting blood glucose (FBG) targets, is an effective and safe option to intensify treatment [5-7]. However, for individuals who cannot achieve HbA1c targets with basal insulin and OADs, questions may arise regarding other treatment options and how to introduce a further-intensified regimen. Furthermore, previous studies demonstrate that adding fast-acting prandial insulin to decrease postprandial blood glucose (PBG) into the normal range is an effective approach $[8,9]$.

Basal insulin plus prandial insulin (basalplus) therapy is considered to be a robust treatment choice, and closely mimics endogenous insulin secretion patterns; it uses a long-acting basal insulin to control FBG and a short-acting prandial insulin bolus to control PBG. However, there are several barriers to intensive basal-plus therapy, including frequent blood glucose monitoring, multiple injections, hypoglycemia, and weight gain, which have a potential negative impact on the acceptance of and compliance to these regimens as well as the quality of life of individuals using them $[10,11]$. Although premixed insulin is one option to avoid some of these barriers, in clinical practice not all patients using premixed insulin can achieve adequate control of blood glucose due to limited doses and the fixed insulin component. In addition, premixed insulins have low flexibility in terms of dose scheduling, which can lead to hypoglycemia in people with more erratic lifestyles and high FBG who do not eat meals at regular times [8].

A previous study found that the addition of one or two boluses of prandial insulin to a regimen of OADs and once-daily basal insulin was effective and more succinct than other options [12]. In addition, observational data from China show basal insulin use is often suboptimal, with relatively low initial doses and inadequate dose titration, and it was hypothesized that optimization of basal insulin through adequate titration before the addition of prandial insulin may be an effective strategy to intensify treatment and improve glycemic control without unnecessarily adding therapeutics and thus increasing the complexity of treatment regimens [13, 14]. However, to the authors' knowledge, no studies conducted in China have examined the efficacy and safety of a treatment strategy involving optimization of basal insulin treatment followed by the addition of prandial insulin. Therefore, this study aimed to investigate the efficacy and safety of intensifying hypoglycemic treatment through the titration of basal insulin followed by the addition of a single bolus of prandial insulin in Chinese people with T2DM suboptimally controlled with basal insulin plus OADs.

\section{METHODS}

\section{Study Design and Subjects}

This is an open-label, single-arm study consisting of a screening phase (up to 2 weeks), insulin glargine dose optimization for 12 weeks (phase I, run-in period), and basal plus prandial insulin treatment for 24 weeks (phase II, intensification period).

Adults aged $30-75$ years with T2DM were recruited at the General Hospital of Tianjin Medical University from March 2015 to July 2015. Eligible subjects had received insulin glargine plus full tolerated doses of two or more OADs for $\geq 6$ months with suboptimal glycemic control (FBG $\geq 6.5 \mathrm{mmol} / \mathrm{L}$ and $\mathrm{HbA} 1 \mathrm{c}>7 \%$ ). Exclusion criteria included type 1 diabetes, current or anticipated pregnancy, treatment with prandial insulin in the past 6 months, or any other multiple insulin injection (i.e., premixed or basal-plus insulin treatments), unconscious hypoglycemia or frequent occurrence of hypoglycemic events, and severe cardiovascular and cerebrovascular diseases or 
other critical diseases. All procedures followed were in accordance with the ethical standards of the responsible committee on human experimentation (institutional and national) and with the Helsinki Declaration of 1964, as revised in 2013. Written informed consent was obtained from all study participants.

\section{Treatment}

Study subjects were initially receiving structured and tailored education based on their lifestyle and dietary habits. All participants received optimization of insulin glargine treatment for 12 weeks during phase I; the insulin glargine (Lantus ${ }^{\circledR}$, Sanofi, France) dose was titrated to obtain an FBG target of $\leq 6.5 \mathrm{mmol} / \mathrm{L}$. At the end of phase I, patients who had achieved $\mathrm{FBG} \leq 6.5 \mathrm{mmol} / \mathrm{L}$ but not $\mathrm{HbA1c} \leq 7 \%$ initiated 24 weeks of prandial insulin (NovoRapid $^{\circledR}$, Novo Nordisk, Denmark) before the main mealtime, adjusted based on the dietary habits of the study subjects. The main meal was defined as the meal resulting in the highest PPG value, determined from a seven-point daytime blood glucose profile (before and $2 \mathrm{~h}$ after each meal and at bedtime) conducted on three separate days in the week prior to initiation of prandial insulin. Following the same methodology as used in the OSIRIS study [12], the initial dose of prandial insulin and dose titration was based on mean PBG, and aimed to achieve a PBG target of $\leq 7.8 \mathrm{mmol} / \mathrm{L} 2 \mathrm{~h}$ after each meal. For safety, insulin secretagogues were discontinued at the addition of prandial insulin and were re-added only in patients with poor glucose control after sufficient insulin titration. OADs were continued as used before entry to the study.

\section{Outcomes}

The primary outcome was change in mean HbA1c from baseline to week 36. Secondary outcomes included the proportion of patients achieving HbA1c $\leq 7 \%$, blood glucose profiles, weight change, and insulin dose. Safety outcomes included incidence of hypoglycemia and other adverse events, including cardiovascular and cerebrovascular events or diarrhea, abdominal distension, dizziness, and vomiting.

\section{Measurements}

Blood glucose levels were measured using the adjusted Contour TS Blood Glucose Meter and Strip (Bayer Pharma, Germany). Patients completed self-blood monitoring four times a day for at least three days per week, and FBG and biochemical parameters were regularly measured in hospital every two weeks. HbA1c was measured throughout the study period using ion-exchange high-performance liquid chromatography (Bio-Rad, USA).

Hypoglycemia was defined as blood glucose $\leq 3.9 \mathrm{mmol} / \mathrm{L}$. Symptomatic hypoglycemia was defined as an event with clinical symptoms consistent with hypoglycemia, with or without a confirmatory blood glucose measurement, and associated with prompt recovery after oral carbohydrate administration. Severe hypoglycemia was defined as an event with symptoms consistent with hypoglycemia in which the patient required assistance, and the event was confirmed by either blood glucose $<2.8 \mathrm{mmol} / \mathrm{L}$ or recovery after oral carbohydrate, intravenous glucose, or glucagon administration.

\section{Statistical Analysis}

All data were analyzed using SPSS Windows version 22.0 (IBM Analytics, USA). Descriptive statistics were used to summarize all data. Quantitative data in a normal distribution were presented as mean (standard deviation [SD]), while enumeration data were presented as a ratio. Changes in parameters between baseline and endpoint were evaluated using a paired-samples $t$-test. An independent $t$-test was used to compare differences in parameters between subgroups, and the $\chi^{2}$ test was used to assess frequency distributions. $p<0.05$ (two tailed) was considered to be statistically significant.

Multiple linear regression analysis was used to identify baseline parameters associated with change in HbA1c at week 36 . The variables 
included in the regression model were: age, height, BMI, disease course, medication time, insulin usage time, basal insulin dosage, baseline $\mathrm{HbA1c}, \mathrm{FBG}$, and PBG.

\section{RESULTS}

\section{Participants and Baseline Characteristics}

A total of 126 individuals were screened and 120 were included in phase I (Fig. 1). At week 12, 110 study subjects had achieved FBG $\leq 6.5 \mathrm{mmol} / \mathrm{L}$, of whom 66 did not achieve $\mathrm{HbA} 1 \mathrm{c} \leq 7 \%$ and were therefore included in phase II. Of the 66 patients included in phase II, three withdrew due to either patient dissatisfaction with treatment outcome $(n=1)$, accidental injury $(n=1)$, or personal reasons $(n=1)$.
At baseline, the mean age of the study subjects was 59.3 (8.7) years, $53.3 \%$ were female, and the mean duration of T2DM was $6.6(2.4)$ years. The mean dose of basal insulin was $0.22 \mathrm{IU} / \mathrm{kg} /$ day, and $98.3 \%, 56.7 \%$, and $74.2 \%$ of subjects were receiving concomitant $\alpha$-glucosidase inhibitors, metformin, and insulin secretagogues, respectively (Table 1). At baseline, mean levels of $\mathrm{HbA1c}$, FBG, and PBG were $8.83 \%, 9.28$, and $14.47 \mathrm{mmol} / \mathrm{L}$, respectively.

\section{Basal Insulin Optimization from Baseline to Week 12}

After 12 weeks of basal insulin optimization, the mean basal insulin dose had significantly increased from $0.22 \mathrm{IU} / \mathrm{kg} /$ day at baseline to $0.36 \mathrm{IU} / \mathrm{kg} /$ day $(p<0.001)$ (Table 1). Between baseline and week 12, a significant decrease in mean HbA1c $(-1.43 \%)$, FBG $(-3.04 \mathrm{mmol} / \mathrm{L})$,

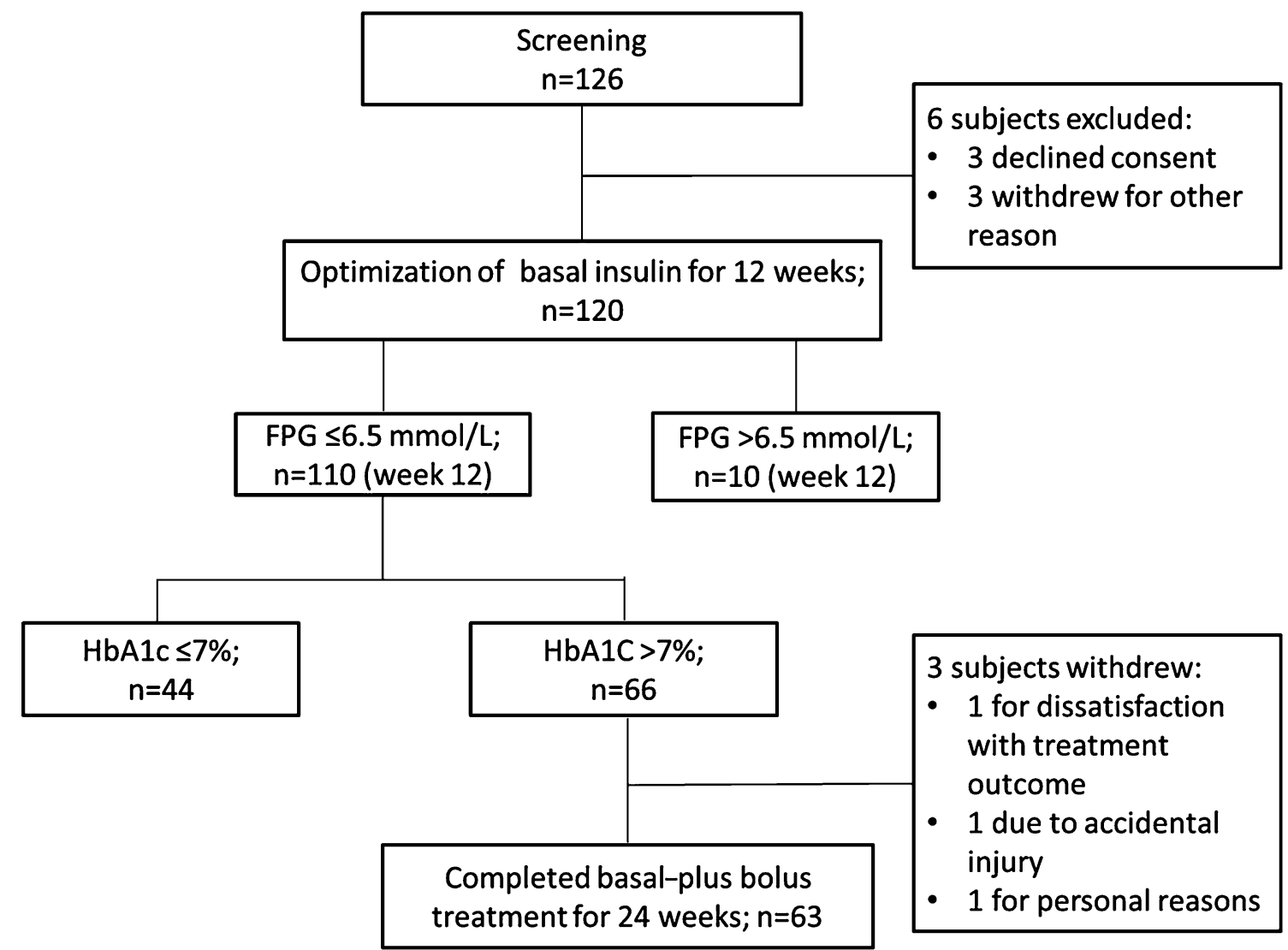

Fig. 1 Study flow diagram. HbAlc glycated hemoglobin A1c 
Table 1 Changes in clinical parameters from baseline to week 12 during basal insulin optimization

\begin{tabular}{lcclr}
\hline Parameters $^{\mathrm{a}}$ & Baseline $(\boldsymbol{n}=\mathbf{1 2 0})$ & Week 12 $(\boldsymbol{n}=\mathbf{1 2 0})$ & Mean difference $(\mathrm{SD})$ & $\boldsymbol{p}$ value \\
\hline Weight $(\mathrm{kg})$ & $71.98(6.39)$ & $72.05(6.38)$ & $0.08(0.81)$ & 0.285 \\
BMI $\left(\mathrm{kg} / \mathrm{m}^{2}\right)$ & $25.82(2.08)$ & $25.85(2.09)$ & $0.03(0.28)$ & 0.271 \\
FBG $(\mathrm{mmol} / \mathrm{L})$ & $9.28(1.34)$ & $6.24(0.29)$ & $3.04(1.32)^{*}$ & $<0.001$ \\
PBG $(\mathrm{mmol} / \mathrm{L})$ & $14.47(2.63)$ & $10.90(2.85)$ & $3.58(1.79)^{*}$ & $<0.001$ \\
HbAlc, $(\%)$ & $8.83(0.84)$ & $7.40(1.04)$ & $1.43(1.01)^{*}$ & $<0.001$ \\
Dose of basal Insulin $(\mathrm{IU} / \mathrm{kg} / \mathrm{day})$ & $0.22(0.05)$ & $0.36(0.06)$ & $0.14(0.04)^{*}$ & $<0.001$ \\
Hypoglycemic events $[n(\%)]$ & $2(1.7)$ & $3(2.5)$ & & 1.000 \\
OADs $[n(\%)]$ & & & & \\
$\alpha$-Glucosidase inhibitor & $118(98.3)$ & $118(98.3)$ & & \\
Metformin & $68(56.7)$ & $68(56.7)$ & & \\
Insulin secretagogues & $89(74.2)$ & $89(74.2)$ & & \\
\hline
\end{tabular}

$B M I$ body mass index, $F B G$ fasting blood glucose, $P B G$ postprandial blood glucose, $O A D s$ oral antidiabetic drugs ${ }^{*} p<0.01$

a Parameters are summarized as mean (SD) unless specified

and PBG (-3.58 mmol/L) was observed (all $p<0.001$ ) (Fig. 2a and b). However, the mean weight and BMI of the study subjects did not change significantly during the 12-week basal insulin optimization phase (Fig. 2c). Hypoglycemia was experienced by three patients during the basal insulin optimization phase (Table 1). The pattern of use of concomitant OADs did not change between baseline and week 12.

\section{Basal Plus Prandial Insulin Treatment from Week 12 to Week 36}

For study subjects who achieved FBG $\leq 6.5 \mathrm{mmol} / \mathrm{L}$ but did not reach $\mathrm{HbA} 1 \mathrm{c} \leq 7 \%$ after 12 weeks of basal insulin optimization, the addition of prandial insulin led to a significant reduction in $\mathrm{HbA} 1 \mathrm{c}$ of $0.89 \%$, with a mean HbA1c of $7.17 \%$ at week $36(p<0.001)$ (Table 2$)$ (Fig. 3a). Furthermore, the mean FBG did not change, and PBG decreased significantly, by $3.03 \mathrm{mmol} / \mathrm{L}(p<0.001)$, following 24 weeks of basal-plus therapy (Fig. 3b). The mean total dose of insulin at week 36 was $0.5 \mathrm{IU} / \mathrm{kg} / \mathrm{day}$. After initiating prandial insulin treatment, $83.1 \%$ of the patients receiving insulin secretagogues ceased using these drugs, and the mean number of OADs used also decreased from 2.71 to 2.12. The proportion of patients completing the 24 weeks of basal- plus treatment was 95.5\% (63/ 66).

Three patients had symptomatic hypoglycemia events during the 24 weeks of basalplus treatment. There was no significant difference in the incidence of hypoglycemia before and after treatment $\left(1.7 \%\right.$ vs. $2.5 \% \chi^{2}=0.21$; $p=1.000$ ) (Table 2 ). No severe hypoglycemia or other severe adverse events were identified during this phase of the study. After 24 weeks of prandial insulin, the mean weight of patients increased slightly without reaching statistical significance versus week 12 (73.2 vs. $73.3 \mathrm{~kg}$; $p=0.379$ ) (Table 2) (Fig. 3c).

\section{Baseline Characteristics of Patients Who Achieved HbA1c $\leq 7 \%$ Versus Those Who Did Not}

The proportion of patients who completed 24 weeks of basal-plus therapy and achieved HbA1c $\leq 7 \%$ was $65.1 \% \quad(41 / 63)$ (Table 3$)$. 
Table 2 Changes in clinical parameters after 24 weeks of basal-plus treatment

\begin{tabular}{lcclr}
\hline Parameters $^{\mathbf{a}}$ & Week 12 $(\boldsymbol{n}=\mathbf{6 3})$ & Week 36 $(\boldsymbol{n}=\mathbf{6 3})$ & Mean difference (SD) & $\boldsymbol{p}$ value \\
\hline HbA1c, $(\%)$ & $8.06(0.71)$ & $7.17(0.54)$ & $0.89(0.56)^{*}$ & $<0.001$ \\
FBG $(\mathrm{mmol} / \mathrm{L})$ & $6.23(0.15)$ & $6.20(0.16)$ & $0.03(0.14)$ & 0.118 \\
PBG $(\mathrm{mmol} / \mathrm{L})$ & $13.17(1.72)$ & $10.14(2.20)$ & $3.03(1.53)^{*}$ & $<0.001$ \\
Basal insulin dosage (IU/kg/day) & $0.36(0.06)$ & $0.35(0.05)$ & $0.01(0.02)$ & 0.073 \\
Prandial insulin dosage (IU/kg/day) & $0(0)$ & $0.14(0.04)$ & $0.14(0.04)^{*}$ & $<0.001$ \\
Total insulin dosage (IU/kg/day) & $0.36(0.06)$ & $0.50(0.08)$ & $0.14(0.03)^{*}$ & $<0.001$ \\
Weight $(\mathrm{kg})$ & $73.19(6.82)$ & $73.28(6.90)$ & $0.08(0.76)$ & 0.379 \\
Number of OADs used & $2.71(0.46)$ & $2.12(0.42)$ & $0.60(0.61)^{*}$ & $<0.001$ \\
OADs, $n$ (\%) & $63(100)$ & $22(34.9)$ & & \\
$\alpha$-Glycosidase inhibitor & $18(28.6)$ & $63(100)$ & & 1.000 \\
Metformin & $59(93.7)$ & $10(15.9)$ & & \\
Insulin secretagogue & $2(1.7)$ & $3(2.5)$ & & \\
Hypoglycemic events $[n(\%)]$ & $0(0)$ & $0(0)$ & & \\
Other adverse events $[n(\%)]$ &
\end{tabular}

$H b A 1 c$ glycated hemoglobin Alc, $F B G$ fasting blood glucose, $P B G$ postprandial blood glucose, $O A D s$ oral antidiabetic drugs ${ }^{*} p<0.01$

a Parameters are summarized as the mean (SD) unless specified

Mean baseline HbA1c level, FBG level, and PBG level were significantly higher among the study subjects who did not achieve HbA1c $\leq 7 \%$ than among those who did achieve this target (differences: 0.94\%, 1.08, and $2.48 \mathrm{mmol} / \mathrm{L}$, respectively; all $p$ values $<0.05$ ) (Table 3). No significant difference in baseline age, disease course, duration of insulin use, baseline insulin dose, weight, height, BMI, or use of OADs was observed between patients who achieved $\mathrm{HbA} 1 \mathrm{c} \leq 7 \%$ and those who did not.

\section{Multiple Linear Regression of Factors Associated with HbA1c at Week 36}

In a stepwise multiple linear regression, baseline HbA1c $(B=0.18, p=0.022)$, PBG $(B=0.15$, $p<0.001)$, and FBG $(B=0.08, p=0.027)$ were significantly associated with HbA1c at the end of phase II (Table 4).

\section{DISCUSSION}

This study demonstrated that optimization of basal insulin treatment followed by the addition of a single bolus of prandial insulin can significantly improve rates of glycemic control in patients with T2DM poorly controlled with basal insulin plus OADs.

Our study found that optimizing basal insulin treatment was effective for increasing rates of glycemic control in patients with T2DM poorly controlled with basal insulin plus OADs, which is consistent with previous research [15]. Forty percent of the study subjects achieved HbA1c $\leq 7 \%$ through a single optimized dose of basal insulin, highlighting the importance of following evidence-based guidelines for basal insulin treatment, which recommend adequate titration to meet FBG targets [2, 3]. In this regard, several large observational studies have shown that treatment with basal insulin in Asia 
Table 3 Differences in baseline parameters between patients who achieved and did not achieve HbAlc $\leq 7 \%$ after 24 weeks of basal-plus treatment

\begin{tabular}{llllr}
\hline Parameters $^{\mathbf{a}}$ & HbA1c $\leq 7 \%(\boldsymbol{n}=\mathbf{4 1})$ & HbA1c $>$ 7\% $(\boldsymbol{n}=\mathbf{2 2})$ & Mean difference $($ SE $)$ & $\boldsymbol{p}$ value \\
\hline Age (years) & $63.76(8.01)$ & $61.64(7.29)$ & $2.12(2.05)$ & 0.306 \\
Disease course (years) & $7.30(2.35)$ & $7.60(2.44)$ & $0.30(0.63)$ & 0.641 \\
Insulin usage time (years) & $2.69(1.70)$ & $2.52(0.92)$ & $0.17(0.39)$ & 0.670 \\
Basal insulin dosage (IU/kg/day) & $0.35(0.06)$ & $0.37(0.06)$ & $0.02(0.02)$ & 0.126 \\
Weight (kg) & $72.71(6.04)$ & $73.73(8.11)$ & $1.02(1.80)$ & 0.574 \\
Height (cm) & $166.34(7.72)$ & $169.09(7.91)$ & $2.74(2.06)$ & 0.187 \\
BMI (kg/m $\left.{ }^{2}\right)$ & $26.28(1.45)$ & $25.39(2.44)$ & $0.89(0.49)$ & 0.076 \\
FBG (mmol/L) & $8.87(1.42)$ & $9.95(1.75)$ & $1.08(0.41)^{*}$ & 0.010 \\
PBG (mmol/L) & $15.01(1.06)$ & $17.49(1.42)$ & $2.48(0.32)^{* *}$ & $<0.001$ \\
HbAlc (\%) & $8.65(0.76)$ & $9.60(0.82)$ & $0.94(0.21)^{* *}$ & $<0.001$ \\
Number of OADs & $2.67(0.48)$ & $2.76(0.44)$ & $0.10(0.14)$ & 0.478 \\
OADs $(n)$ & & & & \\
$\alpha$-Glycosidase inhibitor & 42 & 21 & & \\
Metformin & 10 & 8 & & \\
Insulin secretagogue & 38 & 21 & & \\
\hline
\end{tabular}

$H b A 1 c$ glycated hemoglobin A1c, $B M I$ body mass index, $F B G$ fasting blood glucose, $P B G$ fasting blood glucose, $O A D s$ oral antidiabetic drugs, $S E$ standard error

${ }^{*} p<0.05 ;{ }^{* *} p<0.01$

${ }^{a}$ Parameters are summarized as mean (SD) unless specified

is currently suboptimal, with delayed initiation of treatment and inadequate dose titration $[14,16]$. There are many potential factors leading to this situation, including fear of hypoglycemia, needle anxiety, and worries about weight gain [17], as well as lack of patient education, limited medical resources, inadequate health care systems that make patient follow-up difficult, and suboptimal blood glucose monitoring [18]. The present study shows that when basal insulin is optimized correctly, it can lead to improvements in glycemic control.

There is no clear consensus on the best treatment intensification option for people with T2DM poorly controlled with a combination of basal insulin and OADs; current guidelines recommend the addition of prandial insulin as basal-plus therapy, the use of premixed insulin, or the addition of GLP1 receptor agonists [2, 3]. The results of this study suggest that, for people with T2DM achieving suboptimal glycemic control with basal insulin and OADs, it may be better to first optimize the basal insulin dose to achieve FBG targets by reducing the FBG level to the normal range before considering the addition of other treatments. Indeed, previous research investigating the addition of prandial insulin-either at breakfast or the main mealtime-to basal insulin with OADs for 24 weeks, with no basal insulin optimization phase, reported a mean reduction in HbA1c of $0.4 \%$ [19]. In comparison, the reduction in HbA1c observed with basal-plus in the present study was relatively large $(0.89 \%)$. However, apart from the optimization of basal insulin, there may have been several reasons for this 
Table 4 Multiple linear regression of baseline factors associated with HbAlc after 24 weeks of basal-plus treatment

\begin{tabular}{|c|c|c|c|c|c|}
\hline \multirow[t]{2}{*}{ Parameters } & \multicolumn{2}{|c|}{ Unstandardized coefficient } & \multirow{2}{*}{$\begin{array}{l}\text { Standardized } \\
\text { coefficient }\end{array}$} & \multirow[t]{2}{*}{$T$} & \multirow[t]{2}{*}{$p$ value } \\
\hline & $\bar{B}$ & SE & & & \\
\hline Constant & 3.85 & 1.69 & & 2.28 & 0.027 \\
\hline Age (years) & 0.01 & 0.01 & 0.00 & 0.00 & 0.998 \\
\hline Height (m) & 0.00 & 0.01 & 0.00 & 0.03 & 0.973 \\
\hline BMI $\left(\mathrm{kg} / \mathrm{m}^{2}\right)$ & -0.05 & 0.03 & -0.17 & -1.64 & 0.107 \\
\hline Disease course (years) & -0.01 & 0.03 & -0.06 & -0.50 & 0.624 \\
\hline Medication time (years) & -0.02 & 0.03 & -0.07 & -0.56 & 0.582 \\
\hline Insulin usage time (years) & 0.05 & 0.04 & 0.14 & 1.23 & 0.226 \\
\hline Basal insulin dosage (IU/kg/day) & -0.39 & 0.97 & -0.04 & -0.40 & 0.692 \\
\hline Baseline HbAlc (\%) & 0.18 & 0.08 & 0.30 & 2.36 & $0.022^{*}$ \\
\hline $\mathrm{FBG}(\mathrm{mmol} / \mathrm{L})$ & 0.08 & 0.04 & 0.24 & 2.28 & $0.027^{*}$ \\
\hline $\mathrm{PBG}(\mathrm{mmol} / \mathrm{L})$ & 0.15 & 0.03 & 0.47 & 4.69 & $<0.001^{* *}$ \\
\hline
\end{tabular}

$B M I$ body mass index, $H b A 1 c$ glycated hemoglobin Alc, $F B G$ fasting blood glucose, $P B G$ postprandial blood glucose ${ }^{*} p<0.05 ;{ }^{* *} p<0.01$

difference. Firstly, the diet and exercise of patients in the present study was strictly and comprehensively managed, facilitating the control of PBG and overall blood glucose. Secondly, Chinese people usually eat one large meal during the day. Thirdly, in contrast to the previous study, prandial insulin was tailored based on meal content, which may decrease the level of PBG more effectively and lead to better glycemic control [20,21].

Results from the regression analysis demonstrated that baseline HbA1c, FBG, and PBG were associated with HbA1c levels at week 36, which may aid the selection of patients who are likely to achieve clinical benefit from the treatment approach used in this study. These results were partly consistent with a previous pooled analysis which found that lower baseline HbA1c was the best predictor of whether HbA1c targets would be achieved with insulin glargine and OADs [22]. Results from the present study and those previous findings suggest that individuals with T2DM who have relatively good glycemic control before treatment are more likely to achieve HbA1c targets. An inability to achieve
HbA1c targets may be due to poor self-management in diet and exercise, as well as relatively poor islet cell function [23].

Although the basal-plus treatment approach is well described in the literature and is widely used clinically, this study is, to the author's knowledge, the first investigation of the basal insulin optimization followed by basal-plus approach conducted in China. Given that basal insulin use in China and elsewhere in Asia has been shown to be suboptimal, an investigation of this two-step approach to treatment intensification is worthwhile $[13,14,24]$. In particular, the $40 \%$ of subjects in the present study who achieved HbA1c $\leq 7 \%$ through basal insulin optimization show that the addition of prandial insulin is not necessary for individuals who can derive further benefit from basal insulin.

Several limitations of this study should be noted. Firstly, this was a single-center study, and the results may therefore lack generalizability. Secondly, the patient sample size was relatively small, which limits the strength of the conclusions that can be drawn. Finally, this was a single-arm study, and no control group was 

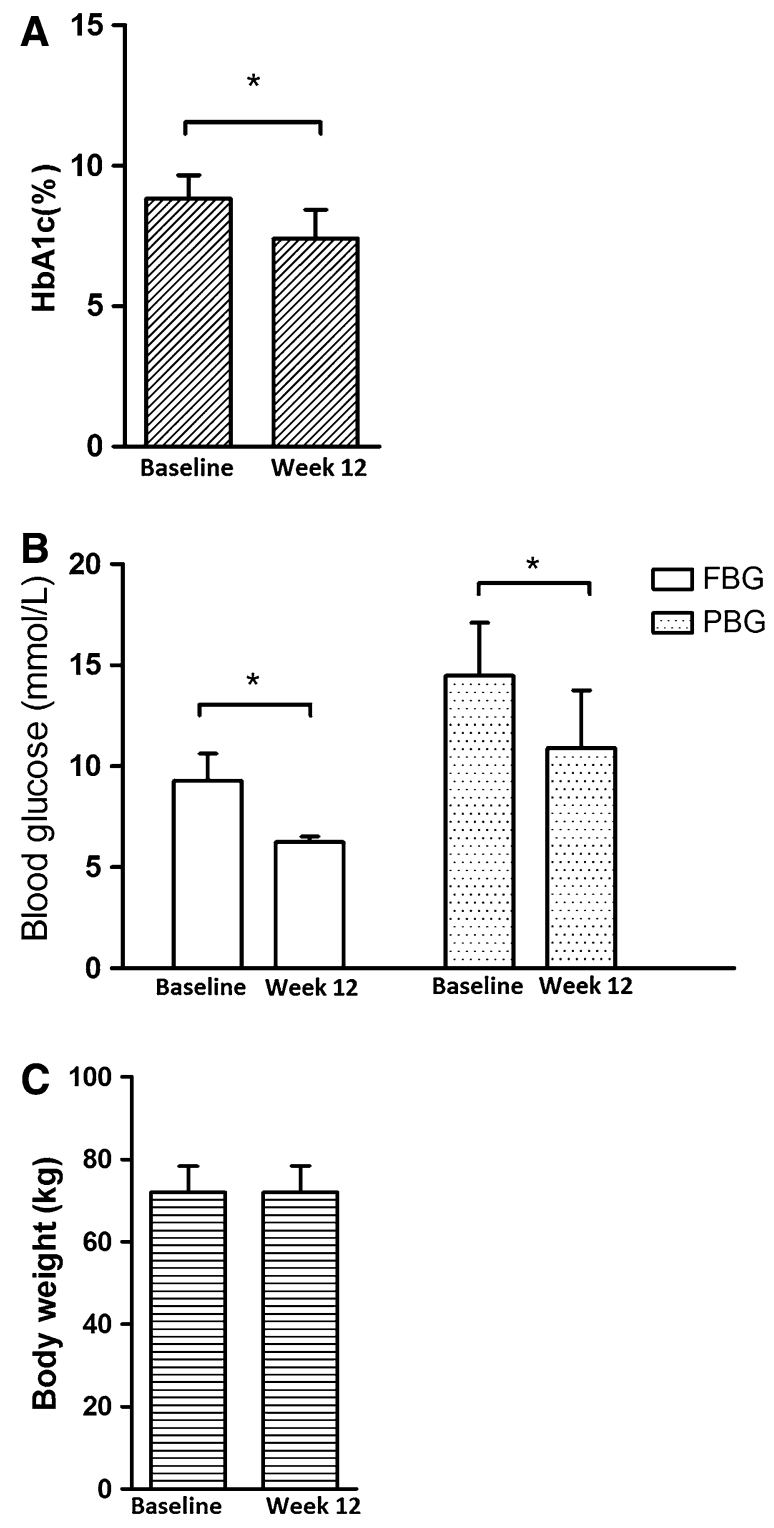

Fig. 2a-c Changes in a HbAlc, b FBG and PBG, and c body weight between baseline and week 12 of basal insulin optimization $(n=120),{ }^{*} p<0.01$

included to allow comparison with other treatment intensification approaches or a placebo. This is a key limitation, as it has been shown that recruitment to a clinical trial, regardless of the intervention, leads to improved glycemic control for individuals with T2DM [25]. As the present study does not include a control arm, it is difficult to separate the improvements in glycemic control ascribable to the investigated
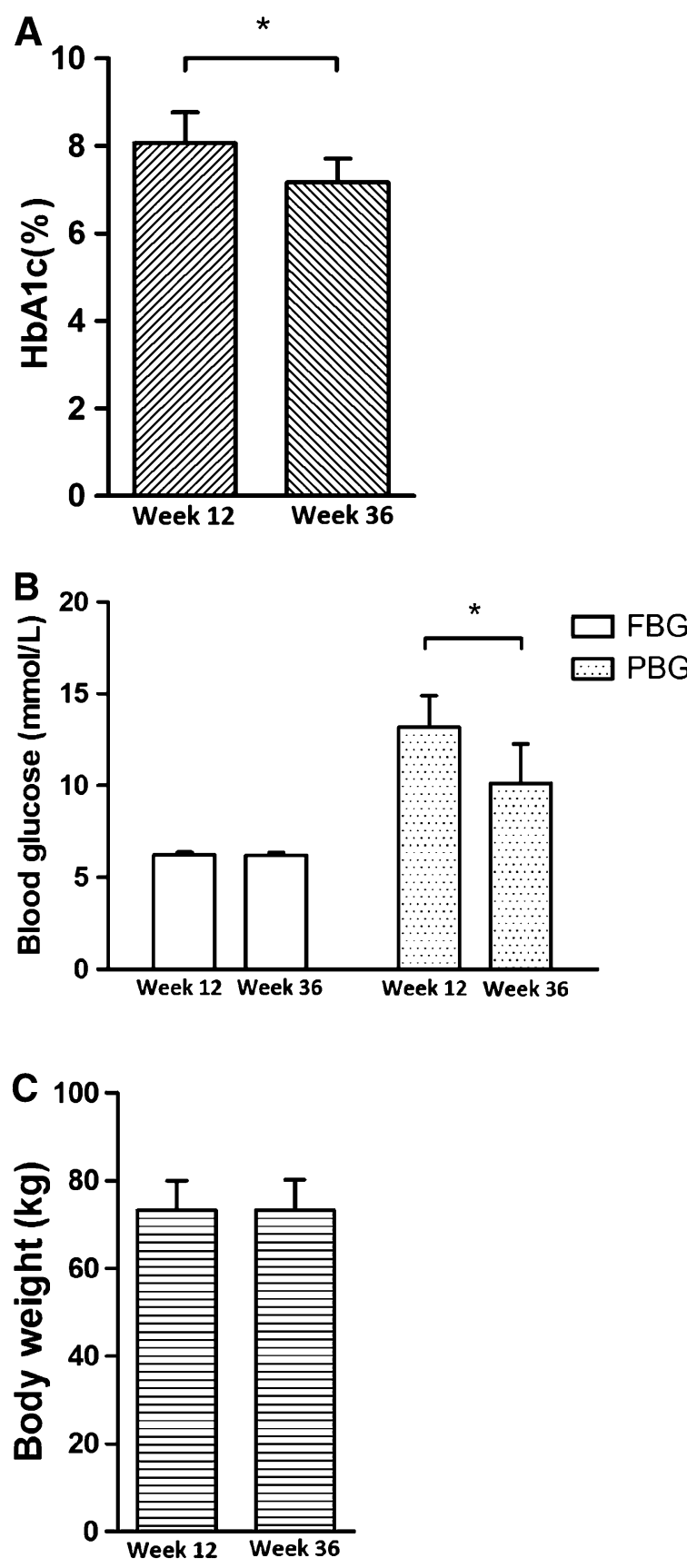

Fig. 3a-c Changes in a HbAlc, b FBG and PBG, and c body weight after 24 weeks' treatment with basal-plus during week 12 to $36(n=63),{ }^{*} p<0.01$

treatment strategy from those gained from entering a clinical trial. However, the reductions in mean HbA1c observed in the present study after 12 weeks of basal insulin 
optimization and after 24 weeks of basal-plus were larger than the reduction in HbA1c previously ascribed to the Hawthorne effect.

\section{CONCLUSION}

In conclusion, basal insulin optimization followed by the addition of a single bolus of prandial insulin may be an effective and safe option for intensifying treatment for people with T2DM poorly controlled with basal insulin and OADs. Large-scale, multicenter, randomized control studies are warranted to validate the results of this study.

\section{ACKNOWLEDGEMENTS}

This study was supported by the Natural Science Foundation of Tianjin City (14JCYBJC24600) and the National Natural Science Foundation of China (H0713:81241030). Editorial support was paid for by Sanofi and provided by Jake Burrell $\mathrm{PhD}$ at Adelphi. No funding was received for article processing charges. All named authors meet the International Committee of Medical Journal Editors (ICMJE) criteria for authorship for this manuscript, take responsibility for the integrity of the work as a whole, and have given final approval of the version to be published. Due to an oversight, this study was not prospectively registered, but the study protocol was reviewed and approved by a local IRB before study initiation. Future clinical trials in this program will be registered where appropriate based on the ICMJE guidelines. Institutional review board/independent ethics committee approval was obtained from Tianjin Medical University General Hospital (IRB2015-YX-010).

Compliance with Ethics Guidelines. All procedures followed were in accordance with the ethical standards of the responsible committee on human experimentation (institutional and national) and with the Helsinki Declaration of 1964, as revised in 2013. Informed consent was obtained from all patients before they were included in the clinical study.
Disclosures. Yujing Jin, Xiaowei Sun, Xichen Zhao, and Tiehong Zhu have no conflict of interest to declare.

Data Availability. The datasets generated during and/or analyzed during the current study are available from the corresponding author on reasonable request.

Open Access. This article is distributed under the terms of the Creative Commons Attribution-NonCommercial 4.0 International License (http://creativecommons.org/licenses/ by-nc/4.0/), which permits any noncommercial use, distribution, and reproduction in any medium, provided you give appropriate credit to the original author(s) and the source, provide a link to the Creative Commons license, and indicate if changes were made.

\section{REFERENCES}

1. International Diabetes Federation. IDF Diabetes Atlas, 7th edn. Brussels, Belgium: International Diabetes Federation. 2015; http://www.idf.org/ diabetesatlas.

2. Garber AJ, Abrahamson MJ, Barzilay JI, et al. Consensus statement by the American Association of Clinical Endocrinologists and American College Of Endocrinology on the Comprehensive Type 2 Diabetes Management Algorithm-2017 Executive Summary. Endocr Pract. 2017;23(2):207-38.

3. Marathe PH, Gao HX, Close KL. American Diabetes Association standards of medical care in diabetes 2017. J Diabetes. 2017;9(4):320-4.

4. Stratton IM, Adler AI, Neil HA, et al. Association of glycaemia with macrovascular and microvascular complications of type 2 diabetes (UKPDS 35): prospective observational study. BMJ. 2000;321(7258):405-12.

5. American Diabetes Association. Standards of medical care in diabetes-2007. Diabetes Care. 2007;30(Suppl 1):S4-41.

6. UK Prospective Diabetes Study (UKPDS) Group. Intensive blood-glucose control with sulphonylureas or insulin compared with conventional treatment and risk of complications in patients with type 2 diabetes (UKPDS 33). Lancet. 1998;352(9131):837-53. 
7. Nathan DM, Buse JB, Davidson MB, et al. Medical management of hyperglycemia in type 2 diabetes: a consensus algorithm for the initiation and adjustment of therapy: a consensus statement of the American Diabetes Association and the European Association for the Study of Diabetes. Diabetes Care. 2009;32(1):193-203.

8. Leahy JL. Basal-prandial insulin therapy: scientific concept review and application. Am J Med Sci. 2006;332(1):24-31.

9. Monnier L, Colette C. Addition of rapid-acting insulin to basal insulin therapy in type 2 diabetes: indications and modalities. Diabetes Metab. 2006;32(1):7-13.

10. Cryer PE, Childs BP. Negotiating the barrier of hypoglycemia in diabetes. Diabetes Spectrum. 2002;15(1):20-7.

11. Meece J. Dispelling myths and removing barriers about insulin in type 2 diabetes. Diabetes Educator. 2006;32(1):S9-18.

12. Raccah D, Haak TJ, Huet D, et al. Comparison of stepwise addition of prandial insulin to a basal-bolus regimen when basal insulin is insufficient for glycaemic control in type 2 diabetes: results of the OSIRIS study. Diabetes Metab. 2012;38(6):507-14.

13. Ji L, Zhang P, Weng J, et al. Observational Registry of Basal Insulin Treatment (ORBIT) in patients with type 2 diabetes uncontrolled by oral hypoglycemic agents in China-study design and baseline characteristics. Diabetes Technol Ther. 2015;17(10):735-44.

14. Tsai ST, Pathan F, Ji L, et al. First insulinization with basal insulin in patients with type 2 diabetes in a real-world setting in Asia. J Diabetes. 2011;3(3): 208-16.

15. Hirsch IB, Bergenstal RM, Parkin CG, Wright E, Buse JB. A real-world approach to insulin therapy in primary care practice. Clin Diabetes. 2005;23(2): 78-86.

16. Kadowaki T, Ohtani T, Naito Y, Odawara M. Potential formula for the calculation of starting and incremental insulin glargine doses: ALOHA subanalysis. PLOS ONE. 2012;7(8):e41358.
17. Korytkowski M. When oral agents fail: practical barriers to starting insulin. Int J Obes Relat Metab Disord. 2002;26(Suppl 3):S18-24.

18. Ji L, Newman J, Lu J, Cai X. Understanding the standard of care in the treatment of type 2 diabetes in China: results from a national survey. Chin Med J (Engl). 2014;127(20):3524-9.

19. Lankisch MR, Ferlinz KC, Leahy JL, Scherbaum WA. Introducing a simplified approach to insulin therapy in type 2 diabetes: a comparison of two single-dose regimens of insulin glulisine plus insulin glargine and oral antidiabetic drugs. Diabetes Obes Metab. 2008;10(12):1178-85.

20. Avignon A, Radauceanu A, Monnier L. Nonfasting plasma glucose is a better marker of diabetic control than fasting plasma glucose in type 2 diabetes. Diabetes Care. 1997;20(12):1822-6.

21. Monnier L, Lapinski H, Colette C. Contributions of fasting and postprandial plasma glucose increments to the overall diurnal hyperglycemia of type 2 diabetic patients: variations with increasing levels of $\mathrm{HbA}(1 \mathrm{c})$. Diabetes Care. 2003;26(3):881-5.

22. Riddle MC, Vlajnic A, Zhou R, Rosenstock J. Baseline $\mathrm{HbA} 1 \mathrm{c}$ predicts attainment of $7.0 \% \mathrm{HbA} 1 \mathrm{c}$ target with structured titration of insulin glargine in type 2 diabetes: a patient-level analysis of 12 studies. Diabetes Obes Metab. 2013;15(9):819-25.

23. Mafauzy M, Hussein Z, Chan SP. The status of diabetes control in Malaysia: results of DiabCare 2008. Med J Malays. 2011;66(3):175-81.

24. Ji L, Zhang P, Zhu D, et al. Observational Registry of Basal Insulin Treatment (ORBIT) in patients with type 2 diabetes uncontrolled with oral antihyperglycaemic drugs: real-life use of basal insulin in China. Diabetes Obes Metab. 2017. [Epub ahead of print].

25. Gale EA, Beattie SD, Hu J, Koivisto V, Tan MH. Recruitment to a clinical trial improves glycemic control in patients with diabetes. Diabetes Care. 2007;30(12):2989-92. 